\title{
Large-scale behavior of the tokamak density fluctuations
}

\author{
G. M. Zaslavsky \\ Courant Institute of Mathematical Sciences, New York University, New York, New York 10012 \\ and Department of Physics, New York University, New York, New York 10003 \\ M. Edelman and $\mathrm{H}$. Weitzner \\ Courant Institute of Mathematical Sciences, New York University, New York, New York 10012 \\ B. Carreras \\ Oak Ridge National Laboratory, Oak Ridge, Tennessee 37831-8070 \\ G. McKee \\ University of Wisconsin/General Atomics, San Diego, California 92186-5608
}

R. Bravenec

University of Texas, Austin, Texas 78712

R. Fonck

University of Wisconsin/General Atomics, San Diego, California 92186-5608

(Received 29 February 2000; accepted 10 May 2000)

\begin{abstract}
An analysis of tokamak density fluctuations data permits the determination of two characteristic exponents. The exponents correspond to the powers of a power-law dependence of the distributions of the long-lasting monotonic change ("flight") of the density and the time length of these changes. Speculation based on these results leads to construction of the fractional kinetic equation for the distribution function of the flights. The asymptotic transport properties of the particle density distribution function are directly connected with the exponents obtained from the density fluctuations data. (C) 2000 American Institute of Physics. [S1070-664X(00)04008-8]
\end{abstract}

\section{INTRODUCTION}

This paper gives an example of how data analysis of the long-time density fluctuations in a fusion-relevant plasma permits the determination of some of its large-scale spatial properties. In order to control a large, hot plasma in a quasistationary regime, one can try to develop a "phenomenology" of its dynamics. In part, the phenomenology should appear as a result of coarse-graining microscopic observations, i.e., small scale space-time processes. Such analysis should lead to the description of some of the large scale space-time processes in terms of small scale processes. Although there is now a rough consensus on what features of the hot plasma should be considered as its large-scale characteristics, we note that small scale phenomena, such as the presence of many magnetic islands, divertor effect, scrapeoff layer physics, etc., can influence the macroscopic dynamics. In line with this approach to the phenomenology we would expect such properties as intermittency and coherent structures to appear in the data. If this goal is realized, the proposed style of analysis would also provide guidance for planning of data acquisition and processing systems for future experiments, so that one might observe correlation between changes of different global properties of fusion plasmas and changes observed by the massive data analysis.

The first question that arises immediately from the preamble is: Is there any indication that from the already known microscopic data there are any large-scale stable characteristics of the tokamak? The purpose of this paper is to answer "yes" to this question. We will address this question by reference to density fluctuation data taken at the plasma core close to the edge. In fact, there can be different scaling ranges which depend not only on where the measurements are performed, but in what regime of the device the data were taken. For example, on the question of the role of internal magnetic islands versus separatrix effects, we might suggest the study of probe measurements within the plasma versus the same fluctuation measurements in the scrape-off layer. Such data are available and could be used for further work. We also mention earlier references on the related issue. $^{1,2}$

It is a common view that the plasma fluctuations in different devices are a result of the complex interaction of nonlinear processes, chaotic dynamics, self-organization, etc., and that these fluctuations may display a self-similar spacetime pattern. Support for this new approach may be found in the data analysis in Refs. 1-3. The so-called self-organized criticality (SOC) concept ${ }^{4}$ has been exploited as a model to represent typical general features of plasma fluctuations. ${ }^{5-8}$ The Hurst exponent ${ }^{9,10}$ has been calculated in Refs. 2 and 3 to describe some fractal properties of the time series of the density fluctuations obtained from different plasma devices. Speculations based on a running sand-pile model were the subject of serious articles, ${ }^{5-8}$ where the large-scale plasma behavior was discussed on the basis of the SOC concept. Although some self-similarity properties observed in the plasma devices are in direct analogy with phenomena of some regimes of sand-piles, this concept by itself does not, at present, lead to a direct determination of a transport model 
applicable to the physical processes in the devices.

An alternate way to examine the self-similarity of the field and density fluctuations is based on the use of models of stochastic processes that are far beyond simple Gaussian processes and that can take into account the effects of memory, intermittency, and fractality in ways that can be explicitly connected to the basic plasma dynamics physics. Examples of such different possibilities of stochastic processes are Lévy-type processes, ${ }^{11}$ Weierstrauss random walks and their modeling of the fractal-time processes, ${ }^{11,12}$ and fractional kinetics phenomenology introduced in Ref. 13 and applied to some dynamical systems. ${ }^{14}$ The essential value in the use of fractional kinetics is the possibility to make an explicit connection between critical exponents of the anomalous transport process and specific features of the dynamics such as "flights" and "trapping time", distributions, both of which are imposed by specific physical phenomena. We include in the term "flights" not only Lévy flights (see, for example, Ref. 11), but also generalized almost-regular bursts in evolution of the system, appearing as a kind of singularity and induced by intermittency, and nearness to a bifurcation point, etc. ${ }^{15}$ Some self-similar regimes of the running sand-pile model can be successfully interpreted from the fractional kinetics point of view. ${ }^{16}$ Not much has been done to explain how the fractional kinetics can be applied straightforwardly to plasma devices. As examples, we mention fractional kinetics of passive particles in the helical flow, ${ }^{17}$ where the hierarchical set of islands in the phase space was pointed out and which defines the anomalous features of the transport; and the attempt (far from being completed) to find a connection between different scales of particle transport and different regimes of the nonlinear dissipative plasma model. ${ }^{18}$

The goal of this paper is not only to show the existence of the self-similarity of the density fluctuations data obtained from the DIII-D tokamak, ${ }^{19}$ but also to emphasize the idea of the necessity of the broad screening of similar data in relation to the manner, specific regime, and specific place of the data acquisition.

\section{ORIGIN OF THE ANOMALOUS PROPERTIES OF FIELDS AND PARTICLE DYNAMICS}

Plasma instabilities are a natural cause of loss fast particles confinement from the volume. Even after averaging over spatial-temporal periods of microoscillations, the evolution of the macroscopic time-space phenomena should appear. Two examples of the origin of the large scale phenomena include the following.

(a) Quasistationary plasmas confined in a threedimensional magnetic configuration possess an underlying magnetic field structure of numerous magnetic islands. Even in a two-dimensional configuration, spontaneous symmetry breaking can lead to a similar situation. ${ }^{20}$ The relative importance of such magnetic structures has been a long-standing controversial issue. ${ }^{21,22}$ However, as more fine-scale electron temperature measurements become available, there is more evidence of magnetic structures in confined plasmas. ${ }^{23,24}$ Detailed information on the nature, volume, and origin of these islands is limited. Nevertheless, on the basis of recent results for the magnetic field lines behavior, we can state that the boundary of an island may be "sticky," i.e., it can attract particle trajectories for some period. This mechanism of the induced ballistic transport differs strongly from the normal, Gaussian type, diffusion. Not all islands are sticky, and those that are sticky generate different transport anomalies. ${ }^{15}$ Independent of the variability of sticky islands, the anomalous transport of field lines or particles can be expressed in the form

$$
\left\langle R^{2}\right\rangle \sim t^{\mu}
$$

with a transport exponent $\mu>1$. This phenomenon is called superdiffusion.

(b) The magnetic field structure at the plasma edge is strongly affected by the presence of a separatrix in magnetic field structure. Thus, a topological effect intervenes in the large-scale particle dynamics through the influence of a specific type of divertor, limiter, etc. Depending on control parameters of the field geometry, ballistic islands may appear in the vicinity of the separatrix ${ }^{25}$ leading to anomalous transport in the poloidal and toroidal directions. Such transport will be reflected in the large scale properties of the distribution function of particles.

(c) Global constraints, such as profile consistency ${ }^{26}$ induced by self-organization dynamics close to a marginal point, can cause an increase in the transport that is characterized by multiple space scales. This phenomenon can also be the cause of anomalous diffusion, exhibited in Eq. (1), which is also contained in the distribution of "flights," and which we introduce later.

Whatever dynamical cause of the anomalous diffusion, the phenomenon may be responsible for some of the experimental observations in magnetically confined plasmas, such as the Bohm scaling of the diffusivities of the low confinement mode and the apparently nonlocal behavior of some perturbative experiments.

From very general considerations one expects that particle density distribution in space and time will have a tail, and that the tail is a complex result of the major large-scale plasma properties. Further, the tail of the distribution, in a simplified version, can be approximated by the distribution function

$$
n_{t}(x, t) \sim t^{\nu_{1}} f\left(x / t^{\nu_{2}}\right) .
$$

The exponents $\nu_{1}, \nu_{2}$ in (2) and $\mu$ in (1) should be coupled to the large scale factors that govern the long-lasting fluctuations, i.e., for example, to the magnetic islands and the fieldlines topology. In fact, expressions (1) and (2) are oversimplified models which can be replaced by a more realistic situation that involve some distribution of values $\mu, \nu_{1}, \nu_{2}$ near their fundamental magnitudes. We discuss this issue further in Sec. VII.

The first step in checking the relevance of this approach to microscopic and macroscopic properties is to analyze the data and to validate the existence of a scaling behavior of the density distribution.

Similarity conditions, such as the one given by Eq. (2), have already been investigated for the probability distribution function of the density fluctuations, plasma potential 
fluctuations, and the induced turbulent particle flux., ${ }^{1,27}$ However, one difficulty often encountered is how to make a reliable determination of the exponents in the tail part of the distribution function of density fluctuations or other extents. The techniques developed here improve the calculation of the exponents over previous determinations.

\section{DESCRIPTION OF THE DATA}

This analysis method is applied to the multichord beam emission spectroscopy (BES) data from the DIII-D tokamak. In the present analysis, we have considered the discharge 92409 for several reasons. This shot was a relatively lowpower negative-shear discharge. For this discharge, the data were collected during its early phase, therefore, there was no $q=1$ surface in the plasma. This eliminates any possible sawtooth oscillations that could add a serious complication in the fluctuation analysis. There was also no evidence in this shot of spurious beam oscillations. Finally, during the time range analyzed, there was no evidence of any coherent mode activity.

The DIII-D BES system has 25 channels, ${ }^{19}$ and gives fluctuation measurements at radial positions separated by about $1 \mathrm{~cm}$. For each channel we have a time record of 250000 points. Data were collected with a sampling rate of 1 MHz. Here, we have considered data from those 6 channels closest to the plasma edge.

Over the shortest time scales, the BES fluctuation measurements are affected by photon noise. This noise is not important for the present studies because our analysis is focused on larger time scales. Furthermore, the analysis shows how we can overcome this contamination. On the other hand, on larger scales, the BES fluctuation measurements can be affected by the so-called common mode, oscillations induced in the beam by the fluctuations at the plasma edge. However, since we are analyzing data relatively close to the plasma edge, it is not necessary in this study to correct for the common mode.

\section{FRACTIONAL EXPONENTS}

We consider a sequence of density fluctuation measurements $\left\{x_{i}\right\}$ at a fixed radial location. We define the cumulative density at a given point in space and at the time $t_{n}$ as

$$
S_{n}=S\left(t_{n}\right)=\sum_{j=1}^{n} x_{j} .
$$

The density $x_{j} \equiv x\left(t_{j}\right)$ is measured at the time $t_{j}$. For large $n=N \gg 1$ the mean square fluctuation is

$$
\left\langle|\delta S(N)|^{2}\right\rangle=\left\langle\left|S_{N}-\left\langle S_{N}\right\rangle\right|^{2}\right\rangle \sim N \text {. }
$$

The mean square fluctuation is proportional to $N$ if the large number law can be applied, or if the sequence $x_{j}$ represents, particularly, Gaussian process. It is known from numerous observations of nonplasma systems that the property (4) can be very poor and sometimes does not reflect specific features of a system and, more specifically, its fractal properties, which typically are attributed to some turbulent process. In general, $S_{n}$ admits fluctuations which do not follow the Gaussian distribution, but which possess power-like tails and infinite high moments.

Let us consider probability distribution function (PDF) $P(\Delta S)$ of fluctuations $\Delta S_{n}$ for different time instants $n$ :

$$
\Delta S_{n} \equiv \Delta S_{n, N}=S_{n}-\left\langle S_{N}\right\rangle n,
$$

where $N$ is the length of the time series and

$$
\left\langle S_{N}\right\rangle=S_{N} / N .
$$

Particularly, for $n=N$ we have simply $\Delta S_{N}=0$. For white noise, $P(\Delta S)$ is Gaussian, while for processes that obey fractional kinetics we expect

$$
P(\Delta S) \sim 1 /|\Delta S|^{\delta} \quad(|\Delta S| \rightarrow \infty) .
$$

So far, the variable $\Delta S$ has not been adequately defined in a way to extract a large scale asymptotics. To do so, we have to consider $\Delta S$ averaged over different time scales. Therefore, let us introduce a coarse-graining of $\Delta S$ corresponding to a "boxing" for the set $\left\{\Delta S_{n}\right\}$ :

$$
\overline{\Delta S_{k}}=(1 / a) \sum_{j=a k+1}^{(k+1) a} \Delta S_{j},
$$

where $a$ is an integer that defines a size of the box. Expression (8) means that instead of the set of $k$ values $\left(\Delta S_{a k+1}, \ldots, \Delta S_{a k+a}\right)$ we consider the mean value $\overline{\Delta S}_{k}$ located at the time interval $(a k+1, a k+a)$.

In Fig. 1 we present the density fluctuation data $\left\{x_{j}\right\}$ for one of the BES channels. The data do not show any significant feature but noise. The situation does not change even after smoothing of $\left\{x_{j}\right\}$ over the nearest 60 points. This is possibly due to the photon noise that is large at small time scales. The pattern becomes different when we consider $\Delta S$, defined in (5), versus $n$ (Fig. 2). A simple look at the new series $\left\{\overline{\Delta S_{k}}\right\}$, which have a length $k_{\max }=N / a$, displays two types of behavior: fairly long-lasting, almost monotonic, segments of the sequence which we will call "flights" and segments with small-scale regular fluctuations. We filter the short-scale fluctuations using a smoothing over the length $a$ and consider only large-scale flights.

Consider an example of a sequence

$$
\begin{aligned}
& \overline{\Delta S}_{k}<\overline{\Delta S}_{k+1}<\ldots<\overline{\Delta S}_{k+m}, \\
& \overline{\Delta S}_{k+m+1}<\overline{\Delta S}_{k+m}, \quad \overline{\Delta S}_{k-1}>\overline{\Delta S}_{k},
\end{aligned}
$$

i.e., the set $\overline{\Delta S}_{j}$ has monotonic changes from $j=k$ to $j=k$ $+m$. We shall call the sequence (9) a flight. Denote $\Delta n_{a}$ to be a length of the monotonic part of $\overline{\Delta S}_{k}$, i.e., the length of a flight. This means that $\Delta n_{a}$ takes values:

$$
\left\{\Delta n_{a}\right\}=\{a, 2 a, 3 a, \ldots\} .
$$

For the example (9), the length of the flight is $\Delta n_{a}=m a$. The flights can be defined in both "directions," i.e., as monotonic increasing or monotonic decreasing of $\overline{\Delta S}_{j}$. Then $P\left(\Delta n_{a}\right)$ is PDF of the length of flights, i.e., time intervals of a monotonic variation of the cumulative density. It will be demonstrated in Sec. $\mathrm{V}$ that $P\left(\Delta n_{a}\right)$ possesses the asymptotic behavior as 

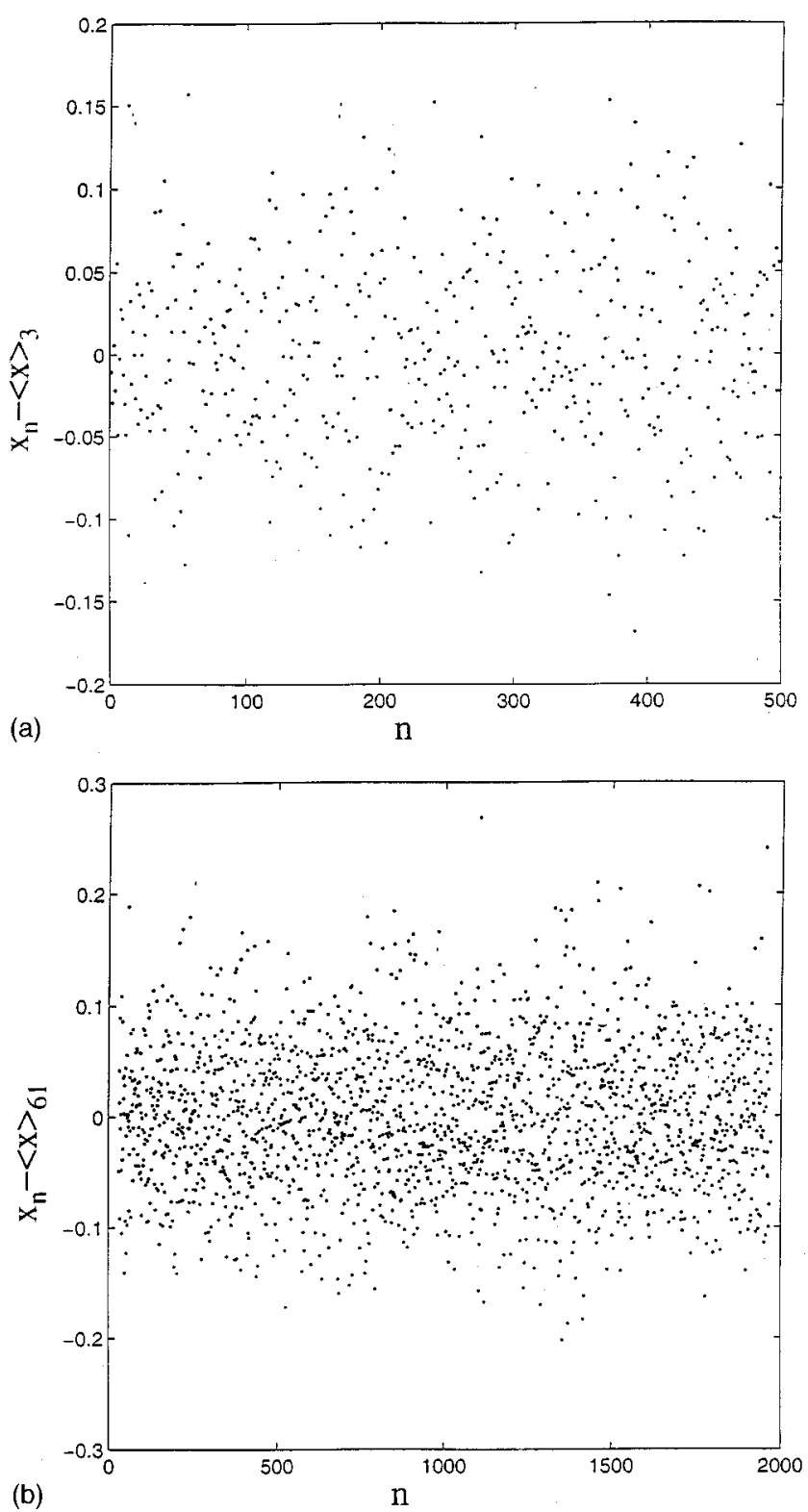

FIG. 1. Two examples of the raw data for density $x_{n}$ at time instant $n$ "smoothed," i.e., averaged over 3 nearest values (a) or over 61 nearest values (b).

$$
P\left(\Delta n_{a}\right) \sim 1 / \Delta n_{a}^{1+\beta}
$$

for some interval of values of $\Delta n_{a}$, and that result is stable with respect to different choices of the value of $a$. In a similar way we may introduce a change of $\overline{\Delta S}$ during the flight, i.e.,

$$
s_{m} \equiv \overline{\Delta S}_{k+m}-\overline{\Delta S}_{k}
$$

for a flight of the length $m$, and consider a PDF $P(s)$ of the cumulative density changes during flights. In other words, $P(s)$ is a distribution function of the cumulative density fluctuations. A specific feature of that density is that we consider only long-lasting fluctuations. It will be seen in Sec. V that

$$
P(|s|) \sim 1 /|s|^{1+\alpha} .
$$

We postpone until later the discussion of the connection between $P(\Delta n)$ and $P(|s|)$.
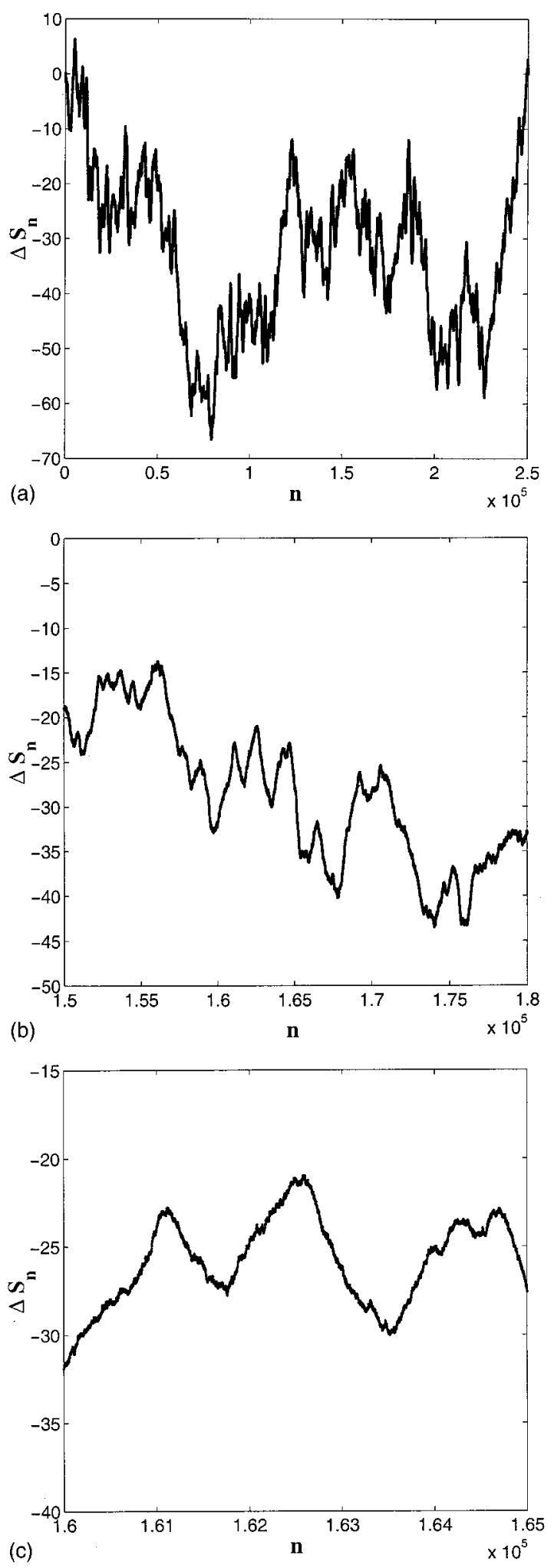

FIG. 2. Values of integral density fluctuation $\Delta S_{n}$ vs time $n$ : raw data (a) and two different zooms (b),(c).

\section{RESULTS FROM THE DATA}

In Fig. 2 we present $\left\{\Delta S_{k}\right\}$ without boxing $(a=1)$. Figure 2 displays numerous flights as well as short-scale noisy parts. Figure 3 presents $P\left(\Delta n_{50}\right)$ and $P\left(\Delta n_{25}\right)$, and these distributions indicate a power law dependence of the tail. 

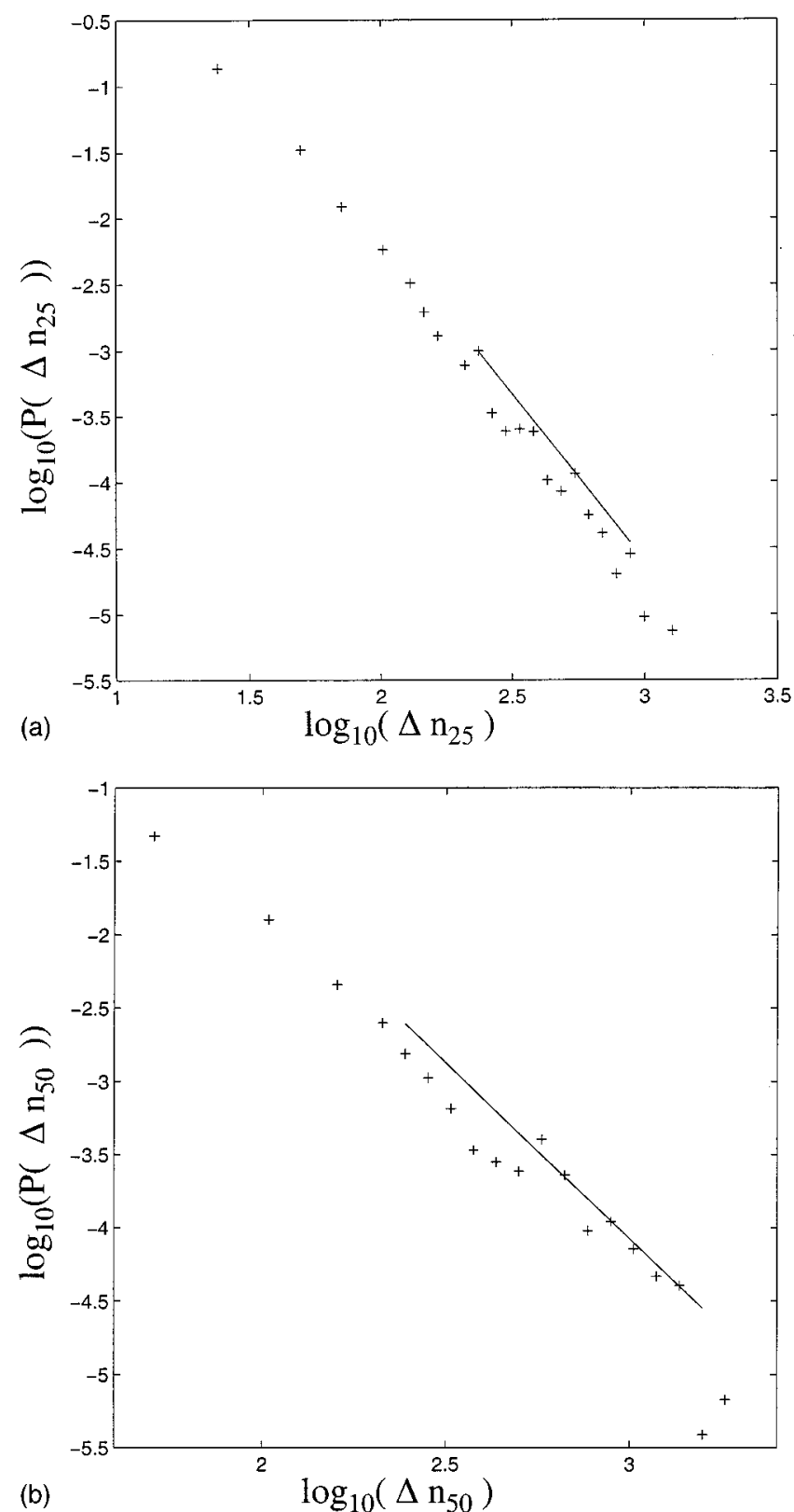

FIG. 3. Distribution of the length of "flights" after coarse graining the data for $\Delta S$ over "boxes" of sizes 25 (a) and 50 (b).

There is no significant difference between $P\left(\Delta n_{50}\right)$ and $P\left(\Delta n_{25}\right)$. The resulting value for $\beta$ in (11) is

$$
\beta=1.4 \pm 0.2 \text {. }
$$

The region of the significant part of the data extends over 1.5 decades, which is a little less than the desirable few decades of data length. Increasing the statistics is thus necessary, and it could be done by selecting data from different shots with similar conditions. Similar behavior with a power-type dependence is obtained for $P(|s|)$ with exponent $\alpha$ within this interval (0.6-1.4) [see Fig. 4 and definition (13)].

This preliminary analysis shows the presence of intermittent behavior which can be induced by a large-scale physical phenomenon such as the effects of magnetic islands or near-separatrix dynamics. Additional information on the

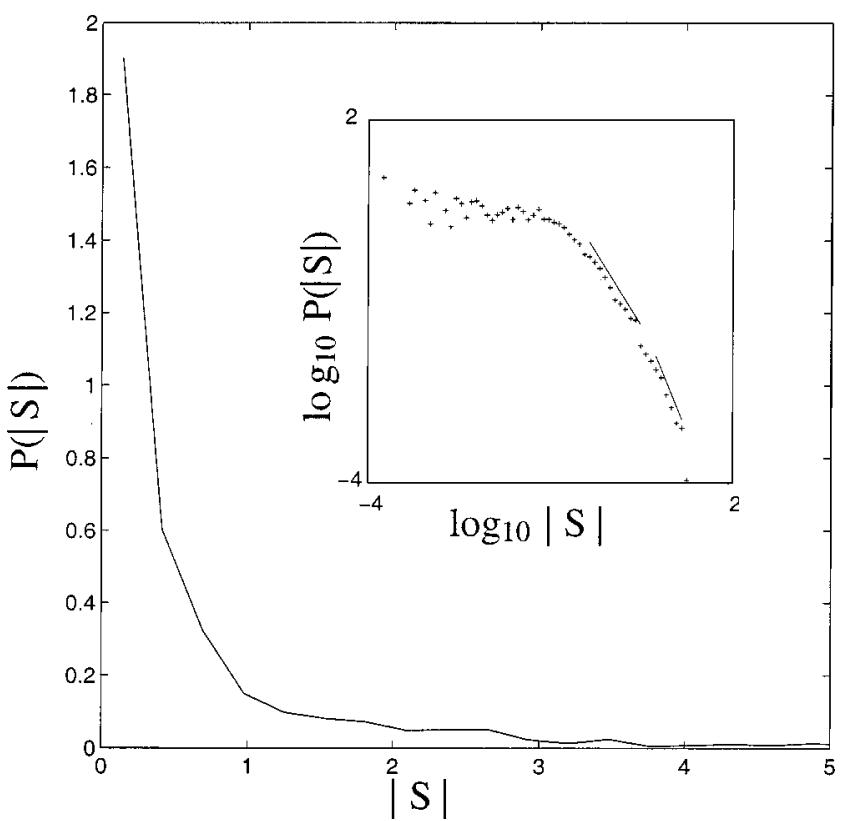

FIG. 4. Distribution of the values of the integral density fluctuations $S=S_{25}$ during one "flight," i.e., large-scale fluctuations.

presence of fractal structures and self-similarities in the data can be obtained by applying a wavelet transform:

$$
\hat{P}(\xi ; b)=\int_{-\infty}^{\infty} d(\Delta n) K(\Delta n-\xi ; b) P(\Delta n)
$$

with a rectangular kernel:

$$
K(\Delta n-\xi ; b)=\frac{1}{2 b}\left\{\begin{array}{rr}
-1, & -b<\Delta n-\xi<0 \\
+1, & 0<\Delta n-\xi<b \\
0, & (\Delta n-\xi)>b
\end{array}\right\}
$$

with the width of the kernel $2 b$. The results are presented in Fig. 5. They indicate the existence of self-similar structures. The largest time interval is $\max \Delta t \sim 75000$; the smallest one is $\min \Delta t \sim 2^{-4} \max \Delta t$. In this way we confirm the existence of the temporal fractal structure in the density dynamics.

\section{DESCRIPTION OF THE FRACTIONAL KINETICS}

Although the data in Figs. 3 and 4 supporting the powerlaw distributions $P(s)$ and $P(n)$ are already significant for understanding the anomalous large scale plasma behavior, the analysis may be much more effective if we can obtain an equation governing the distribution of density fluctuations. Here, we propose to use phenomenological fractional generalization of the Fokker-Planck-Kolmogorov (FFPK) equation, ${ }^{13,14}$ which was successfully applied to some chaotic maps ${ }^{15,28,29}$ as well as to the running sand-pile model. ${ }^{16}$

For this goal, we introduce a distribution function $F_{s}(s, t)$ that describes only a singular part of the full distribution function

$$
F(s, t)=F_{r}(s, t)+F_{s}(s, t),
$$

where $F_{r}(s, t)$ is the regular part of the distribution function. The $F(s, t)$ has a standard meaning as a probability density to have the particle mass $|S|$ at time $t$, while the singular part 


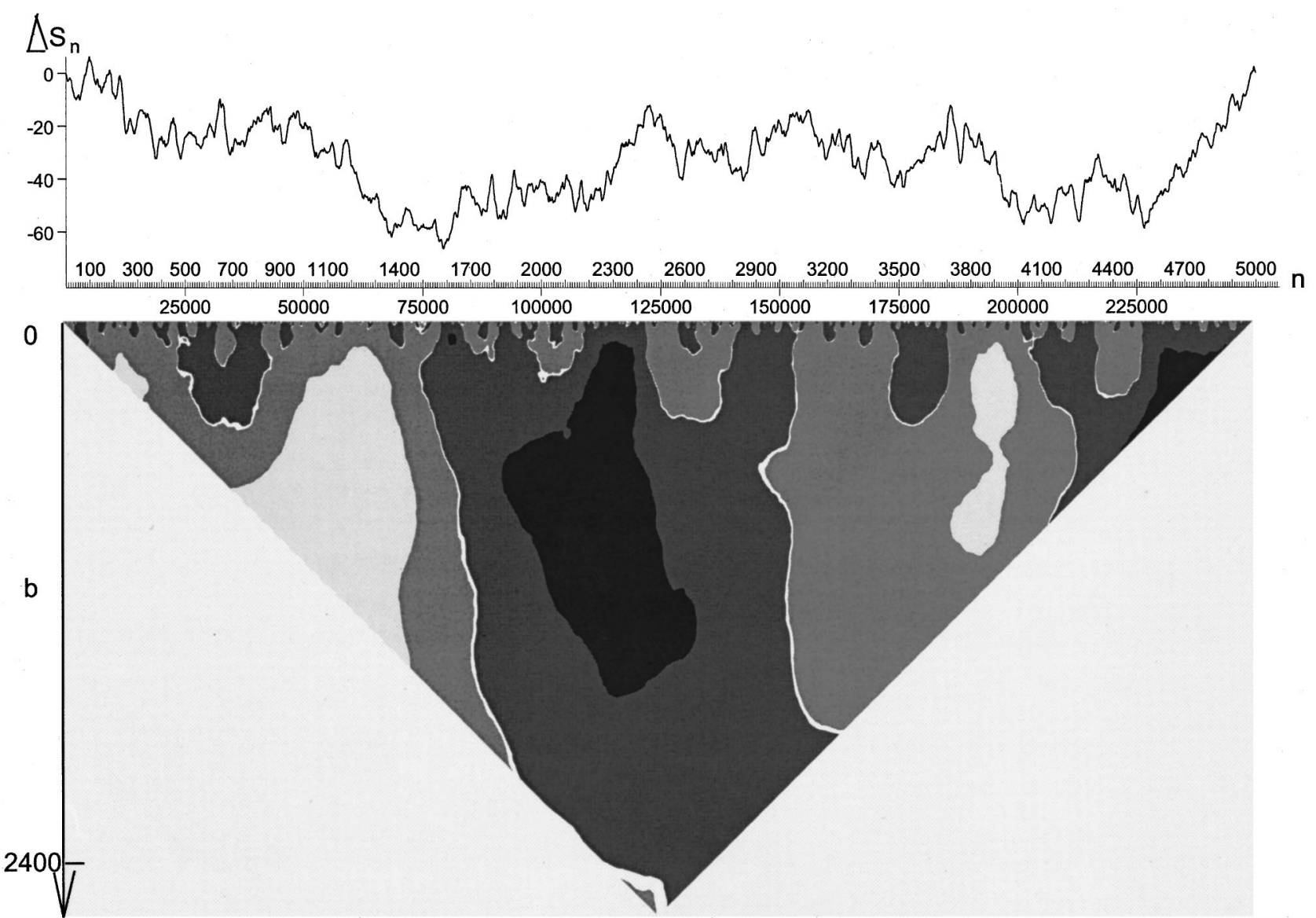

FIG. 5. Wavelet transform $P(\xi, b)$ [see (5.2)] for $b=25$ and for the coarse-grained data $\Delta S_{n}$ [see Fig. 2(a)]. The values of $P$ are in the intervals: ( -1 ; -0.49)—light; $(-0.49 ; 0)$ —grey; $(0 ; 0.49)$ —dark gray; $(0.49 ; 1)$ —black.

$F_{s}(|s|, t)$ describes only the long-lasting and high-magnitude fluctuations of the mass. Then, for a simplified situation to be discussed later, $F_{s}(|s|, t)$ satisfies the FFPK equation

$$
\frac{\partial^{\beta} F_{s}(|s|, t)}{\partial t^{\beta}}=\mathcal{D}_{\alpha \beta} \frac{\partial^{\alpha} F_{s}(|s|, t)}{\partial|s|^{\alpha}}+\mathrm{st},
$$

where $\mathcal{D}_{\alpha \beta}$ is a constant, $\alpha$ and $\beta$ can be fractional, and st is a source term (see more about st in Refs. 30 and 16). Equation (18) has, in particular, self-similar solutions of the type of (2), ${ }^{30}$ and the main problem of finding the solution is to make connection between $\alpha, \beta, \mathcal{D}_{\alpha \beta}$, st, and real physical properties.

Multiplying (18) by $|s|^{\alpha}$ and integrating it over $|s|$, we obtain asymptotically when the st is not important:

$$
\left\langle|s|^{\alpha}\right\rangle=\text { const } t^{\beta}, \quad(t \rightarrow \infty)
$$

and, in a similar way,

$$
\left\langle|s|^{m \alpha}\right\rangle \sim \operatorname{const} t^{m \beta}, \quad(t \rightarrow \infty) .
$$

It is convenient to introduce a transport exponent

$$
\mu=2 \beta / \alpha
$$

which, in the case of a full self-similarity, provides

$$
\left\langle|s|^{2}\right\rangle \sim t^{\mu} .
$$

Expression (22) does not always follow from (19) and (20), especially in the case of multifractality. ${ }^{29}$

Equation (18) without the source term is invariant with respect to the scaling transformation of both variables $|s|$ and $t$. This property can help to establish connection between $\alpha, \beta$ and the observed data (see further details in Refs. 15, 29 , and 16). For the case considered, we may take $\alpha$ and $\beta$ exactly the same as defined in (11) and (13), provided there are no other critical exponents that are important for the particle density evolution. Using (14) and value of $\alpha$ from Fig. 4 , we arrive at

$$
\mu \sim 2.8 \pm 0.8 .
$$

It is a difficulty to define $\mu$ more accurately since the value of $\alpha$, which is close to one, must be determined more precisely.

Let us discuss possible interpretations of the value (23) for $\mu$. In an abstract situation when $s$ is a displacement, $\mu$ $=1$ corresponds to normal diffusion, $1<\mu<2$ means superdiffusion, and $\mu=2$ is ballistic evolution. In the case of (22), $s$ is integral density during its monotonic evolution (i.e., "flight") and the connection between $s$ and particle dynamics is unknown. Due to this, we have an ambiguity in interpretation of (22) and (23) as either a superdiffusion or superballistic regime. The latter case can appear, for example, if 
integral density grows monotonically due to accumulation of the ballistically moving particles, i.e., $|s| \sim l$, where $l$ is a particle displacement. Then the anomalous transport

$$
\langle|s|\rangle \sim t^{1.4 \pm 0.4}
$$

that follows from (22) is the superballistic one or close to it (see also Refs. 31 and 32). That means a local acceleration of particles during a "flight." In fact, the connection $|s| \sim l$ may not exist, and then the superdiffusion will be the major characteristic of the transport process.

\section{DISCUSSION}

We have discussed two issues related to the tokamak data on the density fluctuations obtained from DIII-D in the region close to the plasma edge. The first is, how to work with the data in order to extract large scale density dynamics. It is worthwhile to mention that the raw data are noisy, and the power-law dependencies of the density not appear explicitly. However, we were able to obtain two important characteristics of the density dynamics: power-type distribution of the density fluctuations and a similar behavior of the time length of these fluctuations. We believe that enriched information can be obtained from different regions of plasmas and then compared.

The second issue is related to speculations based on the phenomenologically determined exponents $(\alpha, \beta)$ for the distributions of the integral density fluctuations and their time length. Using the equation of fractional kinetics, it is possible to couple data to the mass/charge transport through the transport exponent $\mu=2 \beta / \alpha$. The result obtained for the square fluctuation of the integral density $s^{2}$ is [see (22)]: $\mu$ $\sim 2.8 \pm 0.8$. At the same time, the Hurst exponent $H$ for the current obtained from tokamaks data in Refs. 1 and 2: $H$ $\sim 0.6-0.75$. A very simplified comparison of the values $\mu$ and $H$ can be obtained in the following way. Let $\hat{s}$ be a density. Then one can write for the second moment

$$
\left\langle s^{2}\right\rangle \sim t^{2}\left\langle\hat{s}^{2}\right\rangle
$$

or, using (22) for the left-hand side, and results of Refs. 1 and 2 for the right-hand side

$$
t^{\mu} \sim t^{2} t^{2 H}
$$

That yields

$$
\mu \sim 2(1+H),
$$

i.e.,

$$
\mu \sim(3.2-3.5)
$$

while our results give

$$
\mu \sim(2-3.6) \text {. }
$$

Since the fairly high error bar for the data (29), one may consider the results (28) and (29) reasonably consistent, although we may expect other situation(s) when more accurate data and better statistics will be used.

This type of study must be extended to include as much fluctuation data as possible. Our results emphasize the im- portance of high accuracy and large statistics for the fluctuation data. We hope that if such data become available, it will not only lead to better information about the core and edge plasma, but will also provide a possibility for precise differentiation of different regimes of tokamak operation. One must note that we have considered the oversimplified situation of pure scaling laws characterized by only two exponents $\alpha$ and $\beta$. In fact, one can expect multifractal situations when the transport formulas (19)-(22) should be improved by introducing multiscaling. We will return to this issue in future publications.

\section{ACKNOWLEDGMENTS}

G.M.Z. was supported by U.S. Department of Energy Grant No. DE-FG02-92ER54184 and by U.S. Navy Grant N00014-96-1-0055. H.W. was supported by U.S. Department of Energy Grant No. DE-FG02-86ER53223. BAC: Research sponsored by Oak Ridge National Laboratory, managed by Lockheed Martin Energy Research Corp. for the U.S. Department of Energy under Contract No. DE-AC0596OR22464.

${ }^{1}$ B. A. Carreras, B. van Milligen, M. A. Pedrosa, R. Balbin, C. Hidalgo, D. E. Newman, E. Sánchez, M. Frances, I. Garcia-Cortés, J. Blueuel, M. Endler, S. Davies, and G. F. Mattews, Phys. Rev. Lett. 80, 4438 (1998).

${ }^{2}$ B. A. Carreras, B. van Milligen, C. Hidalgo, R. Balbin, E. Sánchez, I. Garcia-Cortés, and M. A. Pedrosa, Phys. Rev. Lett. 83, 3653 (1999).

${ }^{3}$ C. Hidalgo, M. A. Pedrosa, B. van Milligen, E. Sánchez, R. Balbin, I. Garcia-Cortés, J. Blueuel, L. Giannone, and H. Niedermeyer, in Fusion Energy 1996 (IAEA, Vienna, 1997), Vol. 1, pp. 617-624.

${ }^{4}$ P. Bak, C. Tang, and K. Weisenfield, Phys. Rev. Lett. 59, 381 (1987).

${ }^{5}$ P. H. Diamond and S. T. Hahm, Phys. Plasmas 2, 3640 (1995).

${ }^{6}$ D. E. Newman, B. A. Carreras, P. H. Diamond, and T. S. Hahm, Phys. Plasmas 3, 1858 (1996).

${ }^{7}$ B. A. Carreras, D. E. Newman, V. E. Lynch, and P. H. Diamond, Phys. Rep. 22, 740 (1996).

${ }^{8}$ X. Garbet and R. Waltz, Phys. Plasmas 5, 2836 (1998).

${ }^{9}$ H. E. Hurst, Trans. Am. Soc. Civil Eng. 116, 770 (1951).

${ }^{10}$ B. B. Mandelbrot and J. W. Wallis, Water Resour. Res. 4, 909 (1969).

${ }^{11}$ E. W. Montroll and M. F. Shlesinger, in Studies in Statistical Mechanics, edited by J. Lebowitz and E. Montroll (North-Holland, Amsterdam, 1984), Vol. 11, p. 1.

${ }^{12}$ B. D. Hughes, E. W. Montroll, and M. F. Shlesinger, J. Stat. Phys. 28, 111 (1982).

${ }^{13}$ G. M. Zaslavsky, in Topological Aspects of the Dynamics of Fluids and Plasmas, edited by H. K. Moffatt, G. M. Zaslavsky, P. Compte, and M. Tabor (Kluwer, Dordrecht, 1992), p. 481.

${ }^{14}$ G. M. Zaslavsky, Physica D 76, 110 (1994); Chaos 4, 25 (1994).

${ }^{15}$ G. M. Zaslavsky, M. Edelman, and B. Niyazov, Chaos 7, 159 (1997).

${ }^{16}$ B. A. Carreras, V. E. Lynch, D. E. Newman, and G. M. Zaslavsky, Phys. Rev. E 60, 4770 (1999).

${ }^{17}$ G. M. Zaslavsky, D. Stevens, and H. Weitzner, Phys. Rev. E 48, 1683 (1993).

${ }^{18}$ S. Benkadda, P. Gabai, and G. M. Zaslavsky, Phys. Plasmas 4, 2864 (1997).

${ }^{19}$ G. McKee, R. Ashley, R. Durst, R. Fobck, M. Jacubovski, K. Tritz, K. Burrell, C. Greenfield, and J. Robinson, Rev. Sci. Instrum. 70, 913 (1999).

${ }^{20}$ B. B. Kadomtsev, Nucl. Fusion 31, 1301 (1991).

${ }^{21}$ J. W. Connor, Plasma Phys. Controlled Fusion 35, 3293 (1993).

${ }^{22}$ R. J. Bickerton, Plasma Phys. Controlled Fusion 39, 339 (1997).

${ }^{23}$ N. J. L. Cardozo, F. S. Shüller, C. J. Barth, C. C. Chu, F. J. Pijper, J. Lok, and A. A. M. Oomens, Phys. Rev. Lett. 73, 256 (1994).

${ }^{24} \mathrm{C}$. Alejaldre and the TJ-II Team, "First Plasmas in the TJ-II Stellarator,", to be published in Nuclear Fusion.

${ }^{25}$ V. Rom-Kedar and G. M. Zaslavsky, Chaos 9, 697 (1999).

${ }^{26}$ B. Coppi, Comments Plasma Phys. Control. Fusion 5, 261 (1980). 
${ }^{27}$ V. Carbone, G. Regnoli, E. Martines, and V. Antoni, Phys. Plasmas 7, 445 (2000).

${ }^{28}$ S. Benkadda, S. Kassibrakis, R. B. White, and G. M. Zaslavsky, Phys. Rev. E 55, 4909 (1997).
${ }^{29}$ G. M. Zaslavsky and M. Edelman, Chaos 10, 135 (2000).

${ }^{30}$ A. I. Saichev and G. M. Zaslavsky, Chaos 7, 753 (1997).

${ }^{31}$ G. Zimbardo, P. Veltri, and P. Pommois, Phys. Rev. E 61, 1940 (2000).

${ }^{32}$ G. Zimbardo, A. Greco, and P. Veltri, Phys. Plasmas 7, 1071 (2000). 